\title{
AUTONOMOUS RESPONSE OF A THIRD-ORDER DIGITAL FILTER WITH TWO'S COMPLEMENT ARITHMETIC REALIZED IN PARALLEL FORM*
}

\author{
BINGO WING-KUEN LING ${ }^{\dagger}$, ALBERT YICK-PO CHAN ${ }^{\dagger}$, THOMAS PAK-LIN WONG $^{\dagger}$, \\ AND PETER KWONG-SHUN TAM ${ }^{\dagger}$
}

\begin{abstract}
This paper investigates the output and state trajectories of a third-order digital filter with two's complement arithmetic realized in parallel form. Although the output of the thirdorder digital filter seems to behave randomly, some regular patterns can be displayed on the plot of $y(k-1)$ versus $y(k)$, where those regular patterns are similar to the second-order case. When the first-order subsystem is operated at the marginally stable points, the output of the third-order system is still mainly dependent on the behaviors of the corresponding second-order digital filter, even though overflow occurs. Explicit equations relating the trajectories of the system to the filter parameters and the initial conditions provide further insights into the behaviors of the system.
\end{abstract}

1. Introduction. It is well known that the autonomous response of a marginally stable second-order digital filter with two's complement arithmetic may exhibit chaotic behaviors, dependent on the initial conditions $[1,4,6,7,10]$. Similar behaviors are reported for the stable and unstable cases $[8,9,12]$. Investigations on the chaotic behaviors of filters with saturation-type nonlinearity and quantization-type nonlinearity are discussed in [2] and [5, 11], respectively.

The step response and sinusoidal response of a marginally stable second-order digital filter with two's complement arithmetic are studied in [13, 14], respectively. It is found that the trajectory of the step response case is similar to the autonomous response case, while that of the sinusoidal response case is more complicated.

In [3], a marginally stable third-order digital filter with two's complement arithmetic implemented in direct form is analyzed. It is found that the trajectory is on some planes of the phase portrait.

In general, a third-order linear digital filter can be realized by a first-order digital filter connected in parallel with a second-order digital filter. However, due to the nonlinearity introduced, one may wonder how would the behaviors of the state variables and the output of the overall system be related to the subsystems. In this paper, we address the following question: How do the state trajectory and the output of the third-order digital filter behave and depend on the filter parameters and the initial conditions of the corresponding first-order and second-order digital filters?

${ }^{*}$ Received on September 13, 2002; accepted for publication on November 28, 2002. This paper is dedicated to Professor John B. Moore on the occasion of his $60^{t h}$ birthday.

${ }^{\dagger} \mathrm{c} / \mathrm{o}$ Department of Electronic and Information Engineering, The Hong Kong Polytechnic University, Hung Hom, Hong Kong. E-mail: bingo@eie.polyu.edu.hk, yick.chan@imperial.ac.uk, thomas.wong@ic.ac.uk, enptam@polyu.edu.hk 
We will review the notations used in the existing literature in section 2 [1-14]. In section 3, some of our results on the autonomous response of a second-order digital filter with two's complement arithmetic [13] are presented. These results are useful for understanding the behavior of a third-order digital filter with two's complement arithmetic realized in parallel form discussed in section 4. Some of other relevant results are presented and discussed in section 5. Finally, a conclusion is summarized in section 6 .

2. Notations. The notations used in [1-14] are adopted as follows:

Assume that a third-order digital filter can be represented by a state space model which realizes parallel connection of a first-order digital filter and a second-order one with two's complement arithmetic in direct form as shown in figure 1. The system can be represented as follows:

$$
\begin{gathered}
{\left[\begin{array}{r}
x_{1}(k+1) \\
x_{2}(k+1)
\end{array}\right]=\left[\begin{array}{c}
x_{2}(k) \\
f\left(b \cdot x_{1}(k)+b \cdot x_{2}(k)+u(k)\right)
\end{array}\right],} \\
y_{1}(k)=x_{1}(k) \\
x_{3}(k+1)=f\left(c \cdot x_{3}(k)+u(k)\right) \\
y_{2}(k)=x_{3}(k) \\
y(k)=f\left(y_{1}(k)+y_{2}(k)\right)
\end{gathered}
$$

where $x_{1}(k), x_{2}(k)$ and $x_{3}(k)$ are the state variables; $u(k)$ is the input signal; $y_{1}(k)$ and $y_{2}(k)$ are the output of the subsystems; $y(k)$ is the output of overall system; $a, b$ and $c$ are the filter parameters; $f$ is the nonlinearity introduced by the two's complement arithmetic.

The nonlinear function $f$ can be modeled as:

$$
f(\nu)=\nu-2 \cdot n \text { such that } 2 \cdot n-1 \leq \nu<2 \cdot n+1 \text { for } n \in Z
$$

Hence,

$$
\begin{array}{r}
{\left[\begin{array}{l}
x_{1}(k) \\
x_{2}(k) \\
x_{3}(k)
\end{array}\right] \in I^{3} \equiv\left\{\left[\begin{array}{l}
x_{1}(k) \\
x_{2}(k) \\
x_{3}(k)
\end{array}\right]:-1 \leq x_{1}(k)<1,\right.} \\
\left.\quad-1 \leq x_{2}(k)<1 \text { and }-1 \leq x_{3}(k)<1\right\} .
\end{array}
$$


In our analysis, we consider the autonomous response, that is:

$$
u(k)=0 \text { for } k \geq 0 \text {. }
$$

Similar to [3], we assume that the corresponding first-order digital filter is stable or marginally stable, and the second-order digital filter is marginally stable, that is:

$$
|c| \leq 1
$$$$
b=-1
$$

and

$$
|a| \leq 2
$$

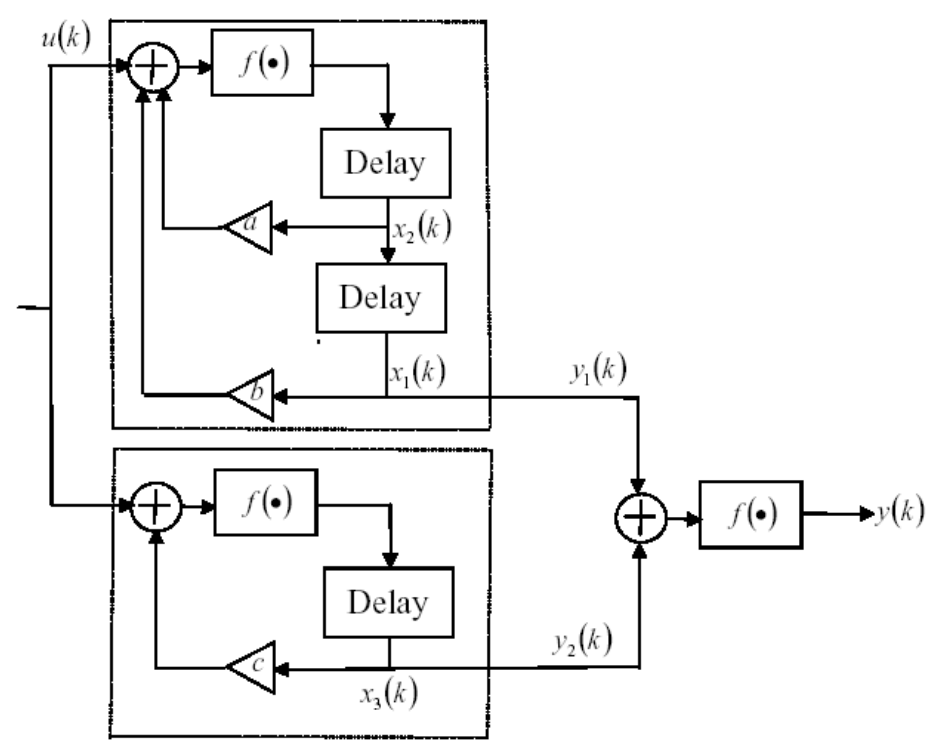

FIG. 1. Parallel realization of a third-order digital filter with two's complement arithmetic.

\section{Review of Some Results on the Autonomous Response of a Second-} order Digital Filter with Two's Complement Arithmetic. If the step size defined in [13] is zero, then the step response of a second-order digital filter with two's complement arithmetic becomes an autonomous response. Hence, our results in [13] apply and we have the following lemmas:

Lemma 1. (L1) Define:

$$
\mathbf{A} \equiv\left[\begin{array}{ll}
0 & 1 \\
b & a
\end{array}\right]
$$




$$
\mathbf{B} \equiv\left[\begin{array}{l}
0 \\
1
\end{array}\right]
$$

and $s_{1}(k)$ such that:

$$
\left[\begin{array}{l}
x_{1}(k+1) \\
x_{2}(k+1)
\end{array}\right] \equiv \mathbf{A} \cdot\left[\begin{array}{l}
x_{1}(k) \\
x_{2}(k)
\end{array}\right]+\mathbf{B} \cdot u(k)+\left[\begin{array}{l}
0 \\
2
\end{array}\right] \cdot s_{1}(k) .
$$

Define

$$
\begin{gathered}
s_{1}^{*} \equiv s_{1}(0), \\
\theta \equiv \cos ^{-1}\left(\frac{a}{2}\right), \\
\hat{\mathbf{A}} \equiv\left[\begin{array}{cc}
\cos \theta & \sin \theta \\
-\sin \theta & \cos \theta
\end{array}\right], \\
\mathbf{T} \equiv\left[\begin{array}{cc}
1 & 0 \\
\cos \theta & \sin \theta
\end{array}\right], \\
\mathbf{x}^{*} \equiv \frac{2 \cdot s_{1}^{*}}{2-a} \cdot\left[\begin{array}{l}
1 \\
1
\end{array}\right], \\
{\left[\begin{array}{cc}
\hat{x}_{1}(k) \\
\hat{x}_{2}(k)
\end{array}\right] \equiv \mathbf{T}^{-1} \cdot\left(\left[\begin{array}{c}
x_{1}(k) \\
x_{2}(k)
\end{array}\right]-\mathbf{x}^{*}\right) \text { for } k \geq 0 .}
\end{gathered}
$$

The following three statements for the type I trajectory are equivalent:

$$
\begin{aligned}
& (L 1.1) \quad\left[\begin{array}{l}
\hat{x}_{1}(k+1) \\
\hat{x}_{2}(k+1)
\end{array}\right]=\hat{\mathbf{A}} \cdot\left[\begin{array}{l}
\hat{x}_{1}(k) \\
\hat{x}_{2}(k)
\end{array}\right] \text { for } k \geq 0, \\
& (L 1.2) \quad s_{1}(k)=s_{1}^{*} \text { for } k \geq 0
\end{aligned}
$$

and

$$
\begin{aligned}
(L 1.3) & {\left[\begin{array}{l}
x_{1}(0) \\
x_{2}(0)
\end{array}\right] \in \Xi_{0} } \\
\equiv & \left\{\left[\begin{array}{l}
x_{1}(0) \\
x_{2}(0)
\end{array}\right]:\left\|\mathbf{T}^{-1} \cdot\left(\left[\begin{array}{l}
x_{1}(0) \\
x_{2}(0)
\end{array}\right]-\mathbf{x}^{*}\right)\right\| \leq 1-\left|\frac{2 \cdot s_{1}^{*}}{2-a}\right|\right\} .
\end{aligned}
$$

Lemma 2. (L2): Suppose $\theta$ is not an integral multiple of $\pi$. Define:

$$
\mathbf{x}_{0}^{*} \equiv\left(\mathbf{I}-\mathbf{A}^{M}\right)^{-1} \cdot \sum_{j=0}^{M-1} \mathbf{A}^{M-1-j} \cdot\left[\begin{array}{l}
0 \\
2
\end{array}\right] \cdot s_{1}(j),
$$




$$
\mathbf{x}_{i+1}^{*} \equiv \mathbf{A} \cdot \mathbf{x}_{i}^{*}+\left[\begin{array}{l}
0 \\
2
\end{array}\right] \cdot s_{1}(i) \text { for } i=0,1, \cdots, M-2
$$

and

$$
\hat{\mathbf{x}}_{i}(k) \equiv \mathbf{T}^{-1} \cdot\left(\left[\begin{array}{c}
x_{1}(k \cdot M+i) \\
x_{2}(k \cdot M+i)
\end{array}\right]-\mathbf{x}_{i}^{*}\right) \text { for } k \geq 0 \text { and } i=0,1, \cdots, M-1 .
$$

The following three statements for the type II trajectory are equivalent:

$$
(L 2.1) \quad \hat{\mathbf{x}}_{i}(k+1)=\hat{\mathbf{A}}^{M} \cdot \hat{\mathbf{x}}_{i}(k) \text { for } k \geq 0 \text { and } i=0,1, \cdots, M-1,
$$

$$
(L 2.2) s_{1}(k \cdot M+i)=s_{1}(i) \text { for } k \geq 0 \text { and } i=0,1, \cdots, M-1
$$

and

$$
\begin{array}{r}
(L 2.3)\left[\begin{array}{l}
x_{1}(0) \\
x_{2}(0)
\end{array}\right] \in \Xi_{1} \equiv\left\{\left[\begin{array}{l}
x_{1}(0) \\
x_{2}(0)
\end{array}\right]:\left\|\mathbf{T}^{-1} \cdot\left(\left[\begin{array}{l}
x_{1}(i) \\
x_{2}(i)
\end{array}\right]-\mathbf{x}_{i}^{*}\right)\right\|\right. \\
\left.\leq 1-\left\|\mathbf{x}_{i}^{*}\right\|_{\infty}\right\} \text { for } i=0,1, \cdots, M-1 .
\end{array}
$$

Lemma 3. (L3) Define:

$$
\Xi_{2} \equiv I^{2} \backslash\left(\Xi_{0} \cup \Xi_{1}\right)
$$

where $I^{2} \equiv\left\{\left[\begin{array}{l}x_{1}(k) \\ x_{2}(k)\end{array}\right]:-1 \leq x_{1}(k)<1\right.$ and $\left.-1 \leq x_{2}(k)<1\right\}$. The following three statements for the type III trajectory are equivalent:

and

$$
(L 3.3)\left[\begin{array}{l}
x_{1}(0) \\
x_{2}(0)
\end{array}\right] \in \Xi_{2}
$$

The proofs can be found in [13]. It can be seen that some initial conditions which are very close in numerical values may give rise to quite different system trajectories $[1,13]$. Figures $2 \mathrm{a}, 2 \mathrm{~b}$, and $2 \mathrm{c}$ show some typical state trajectories corresponding to the type I, type II, and type III trajectories, respectively. Lemmas L1.1, L2.1 and L3.1 give analytical expressions for the different types of trajectories, while Lemmas L1.3, L2.3 and L3.3 give analytical expressions for the corresponding sets of initial conditions. 


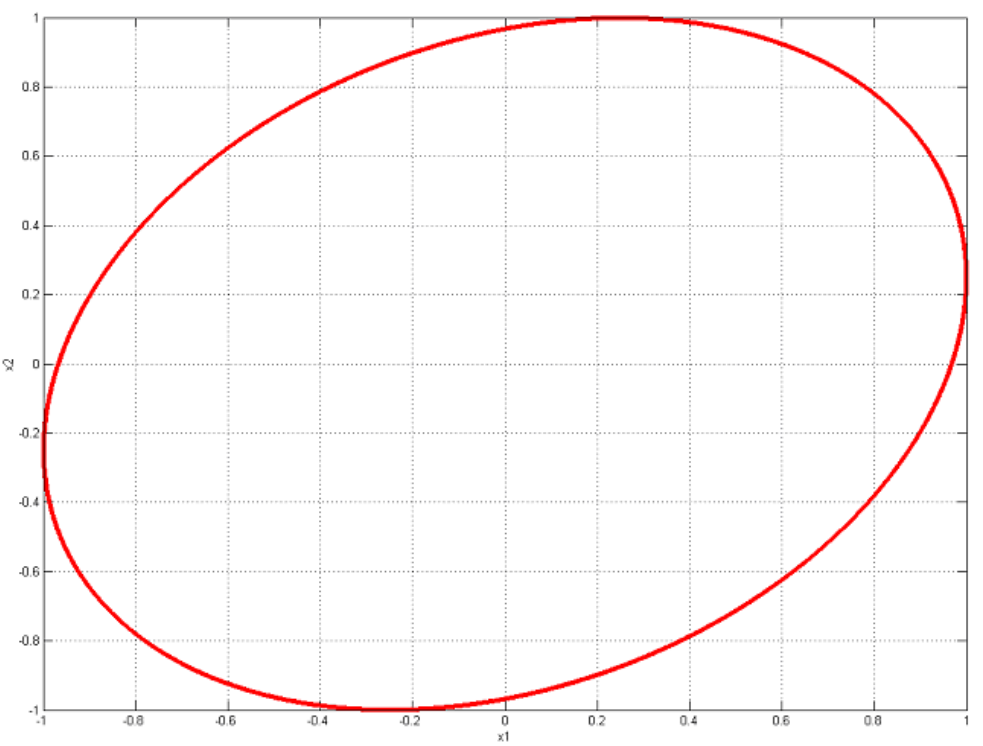

FIG. 2a. Type I trajectory with $a=0.5$ and $\mathbf{x}(0)=\left[\begin{array}{c}0.612 \\ -0.612\end{array}\right]$.

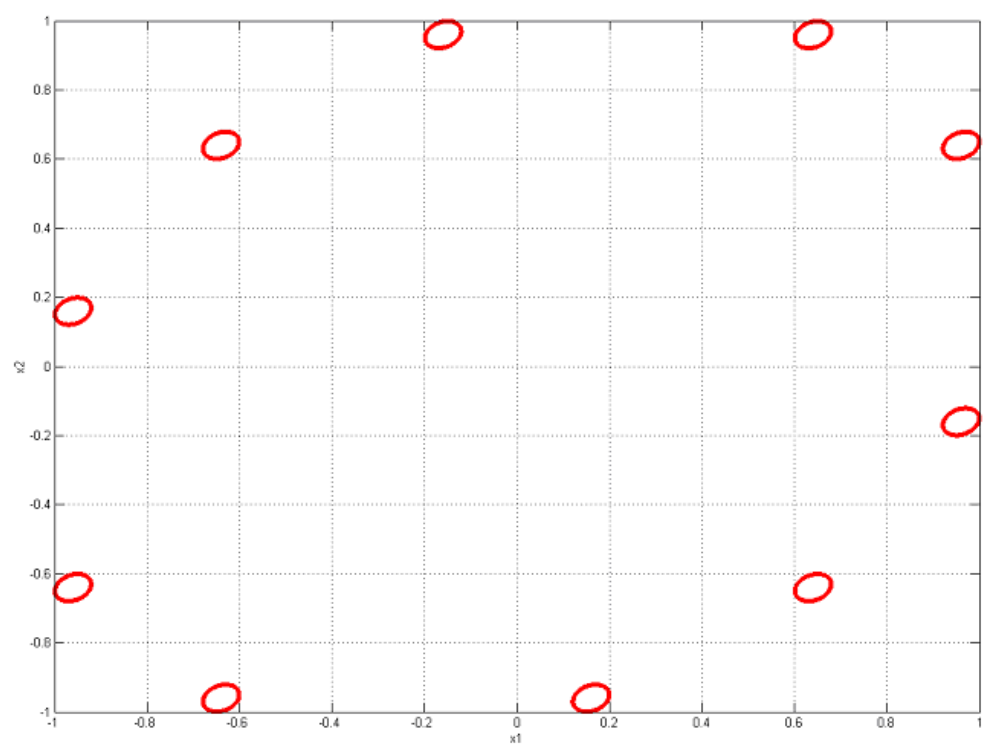

FIG. 2b. Type II trajectory with $a=0.5$ and $\mathbf{x}(0)=\left[\begin{array}{c}0.616 \\ -0.616\end{array}\right]$.

4. Analytical and Simulation Results. In this section, we will analyze the state trajectories and the output of the system described in section 2 by considering the following three cases: $|c|<1, c=1$ and $c=-1$, respectively.

4.1. $|c|<1$. 


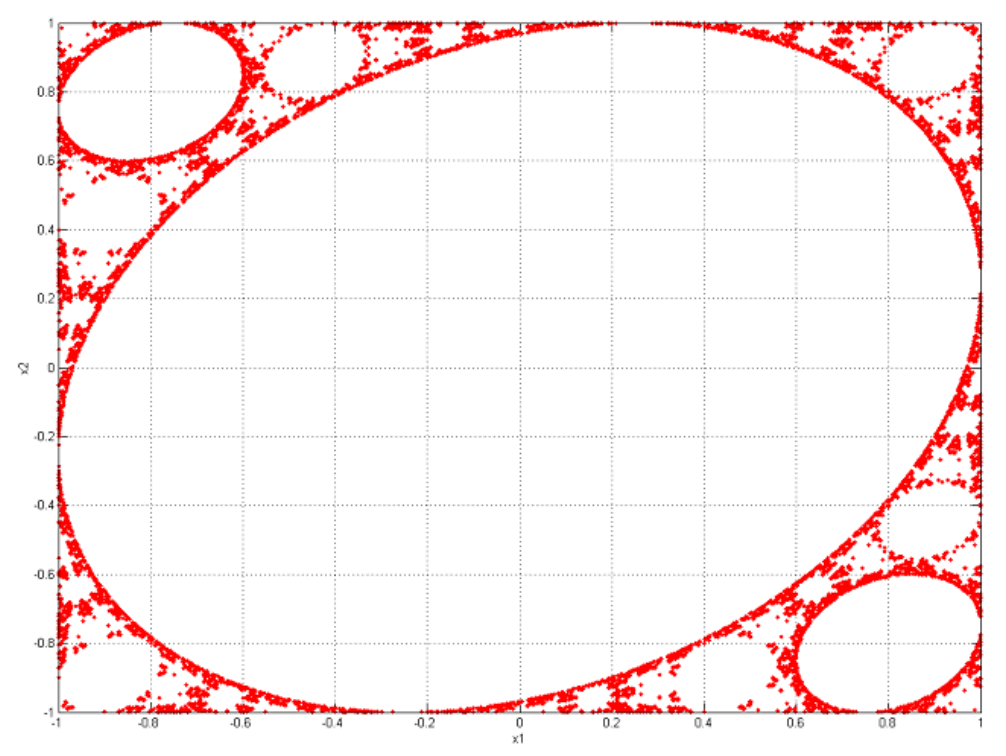

FIG. 2c. Type III trajectory with $a=0.5$ and $\mathbf{x}(0)=\left[\begin{array}{c}0.6135 \\ -0.6135\end{array}\right]$.

We note that if $|c|<1$, the state trajectory converges to the plane $x_{3}=0$ for all initial conditions $x_{3}(0) \in[-1,1)$. Hence, the trajectories are attracting. The plot of the output $y(k-1)$ versus $y(k)$ exhibits three types of trajectories, depended on the initial condition of the corresponding second-order subsystem. If the system exhibits type I or type II trajectories, the trajectories on the elliptical orbits of the plane are dense if $\theta$ is not a rational multiple of $\pi$. For the type III trajectory, the trajectory on the fractal region of the plane is also dense. To illustrate the results, we have performed some computer simulations using the software MATLAB in PC. All calculations are carried out with a 64-bit format. Although we assume that the state variables can be taken any values between -1 and 1 , and infinite word length should be used for the representation of the state variables, some researchers reported that 16 bits are enough for the representation $[5,11]$. Hence, our simulations give the reasonable predications of the system behaviors. In the simulation, $c=0.99$ and $a=0.5$. The initial condition $x_{3}(0)$ is generated randomly in $[-1,1)$. Figures $3 \mathrm{a}, 3 \mathrm{~b}$, and $3 \mathrm{c}$ show the state trajectories corresponding to the type I, type II, and type III trajectories, respectively. And figures $4 \mathrm{a}, 4 \mathrm{~b}$, and $4 \mathrm{c}$ show the corresponding plot of the output $y(k-1)$ versus $y(k)$.

\section{2. $c=1$.}

For $c=1$, the first-order subsystem is operating at the margin of stability and its output does not converge to zero. Hence, overflow may occur when the output of the corresponding first-order digital filter is added to that of the corresponding 


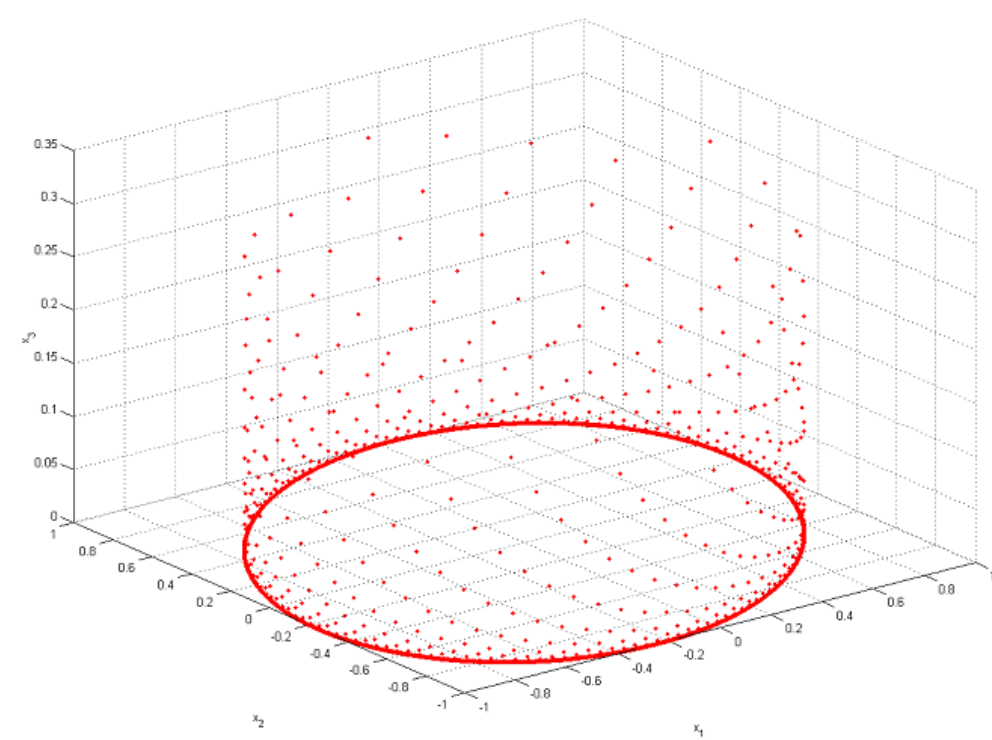

FIG. 3a. Type I trajectory with $c=0.99, a=0.5$ and $\mathbf{x}(0)=\left[\begin{array}{c}0.612 \\ -0.612 \\ 0.3\end{array}\right]$.

second-order digital filter.

The state trajectories stay on a plane with $x_{3}=x_{3}(0)$. In this case, there are no attracting or repelling regions for the trajectories. There are again three types of trajectories, depending on the initial conditions of the corresponding second-order subsystem, as shown in figures $5 \mathrm{a}, 5 \mathrm{~b}$ and $5 \mathrm{c}$, respectively. If the system exhibits type I or type II trajectories, the trajectories on the elliptical orbits of the plane are dense if $\theta$ is not a rational multiple of $\pi$. For the type III trajectory, the trajectory on the fractal region of the plane is also dense.

As $y_{1}(k)=x_{1}(k)$ for $k \geq 0$ and $y_{2}(k)=x_{3}(0)$, for $k \geq 0$, we have $y(k)=$ $f\left(y_{1}(k)+x_{3}(0)\right)$. In order to understand the plot of $y(k-1)$ versus $y(k)$, let's consider the plot of $y_{1}(k-1)+x_{3}(0)$ versus $y_{1}(k)+x_{3}(0)$. As the plot of $y_{1}(k-1)$ versus $y_{1}(k)$ is the trajectory of the corresponding second-order digital filter, the plot of $y_{1}(k-1)+x_{3}(0)$ versus $y_{1}(k)+x_{3}(0)$ is to shift the trajectory of the corresponding second-order digital filter both horizontally and vertically by $x_{3}(0)$, as shown in figure 6 . Hence, the plot of $y(k-1)$ versus $y(k)$ is obtained by applying the two's complement operation on the shifted trajectory of the corresponding second-order digital filter, both horizontally and vertically. For example, consider the case when $x_{3}(0)>0$, if $y_{1}(k-1)+x_{3}(0) \geq 1$ and $-1 \leq y_{1}(k)+x_{3}(0)<1$, that is, the point is in the black region in figure 6 , then $y(k-1)=y_{1}(k-1)+x_{3}(0)-2$ and $y(k)=y_{1}(k)+x_{3}(0)$, which is moved to the region in figure 6 filled by spare dots. Similar results are obtained for $y_{1}(k)+x_{3}(0) \geq 1$ and $-1 \leq y_{1}(k-1)+x_{3}(0)<1$, 


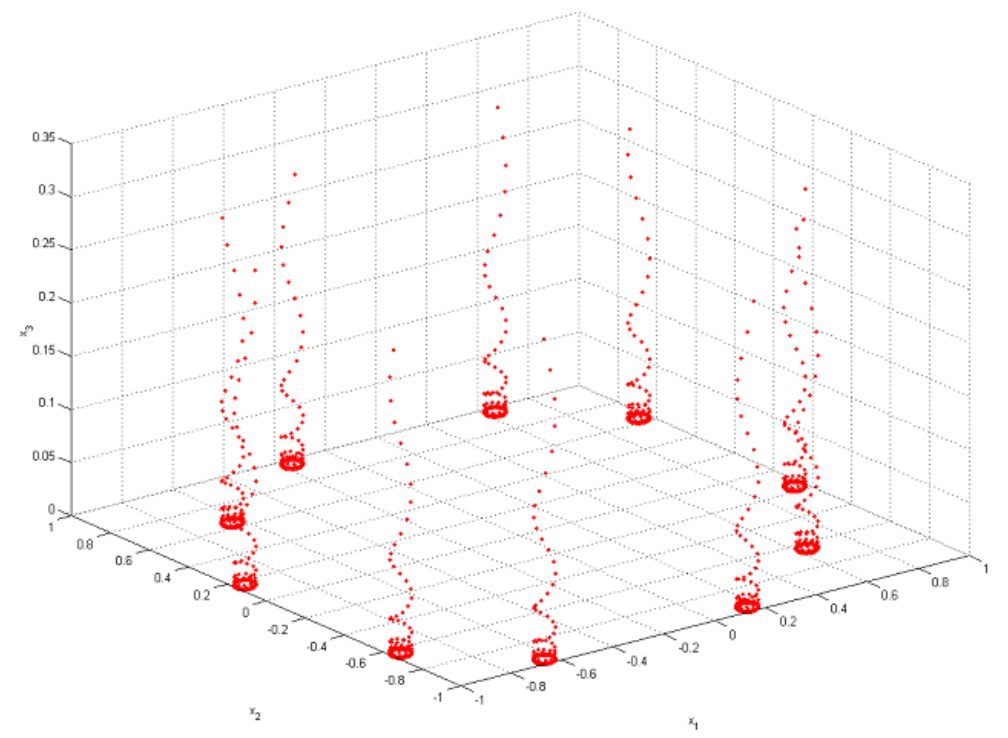

FIG. 3b. Type II trajectory with $c=0.99, a=0.5$ and $\mathbf{x}(0)=\left[\begin{array}{c}0.616 \\ -0.616 \\ 0.3\end{array}\right]$.

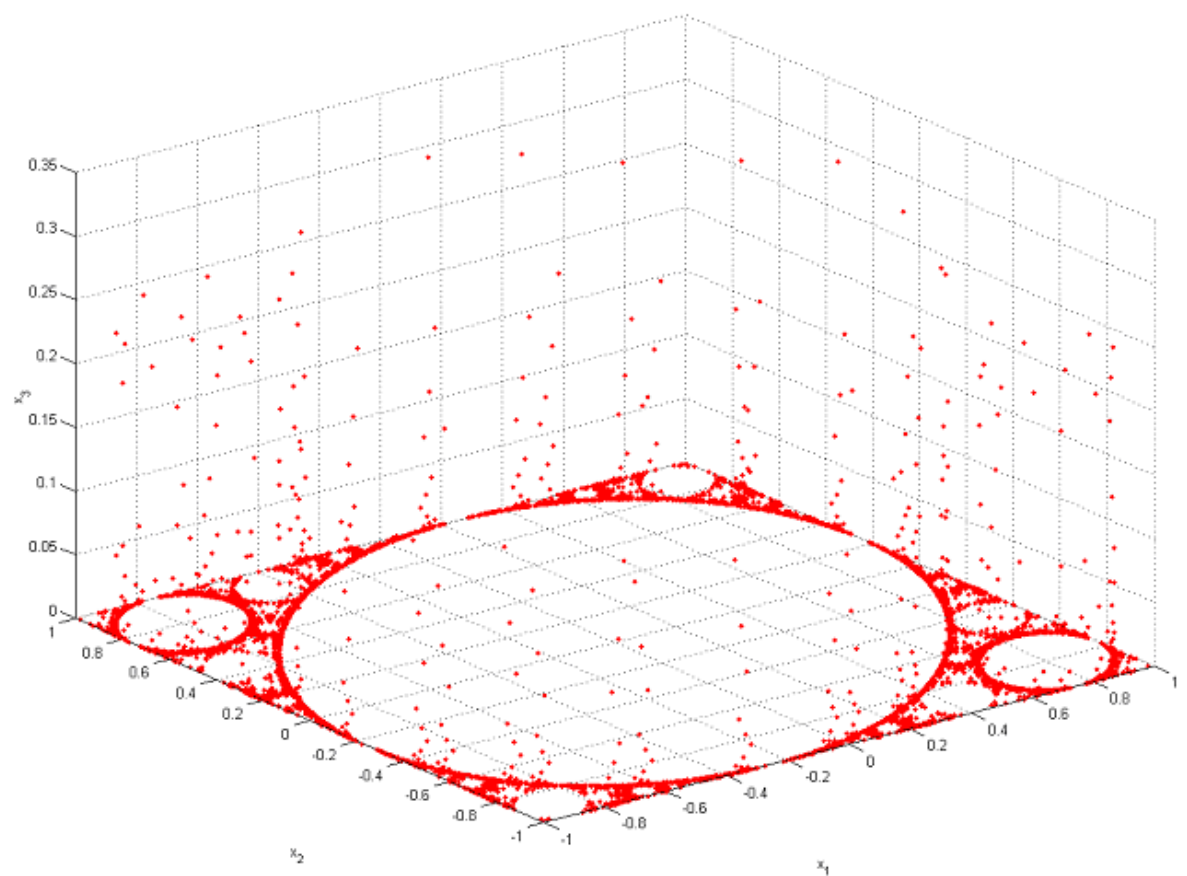

FIG. 3c. Type III trajectory with $c=0.99, a=0.5$ and $\mathbf{x}(0)=\left[\begin{array}{c}0.6135 \\ -0.6135 \\ 0.3\end{array}\right]$. 


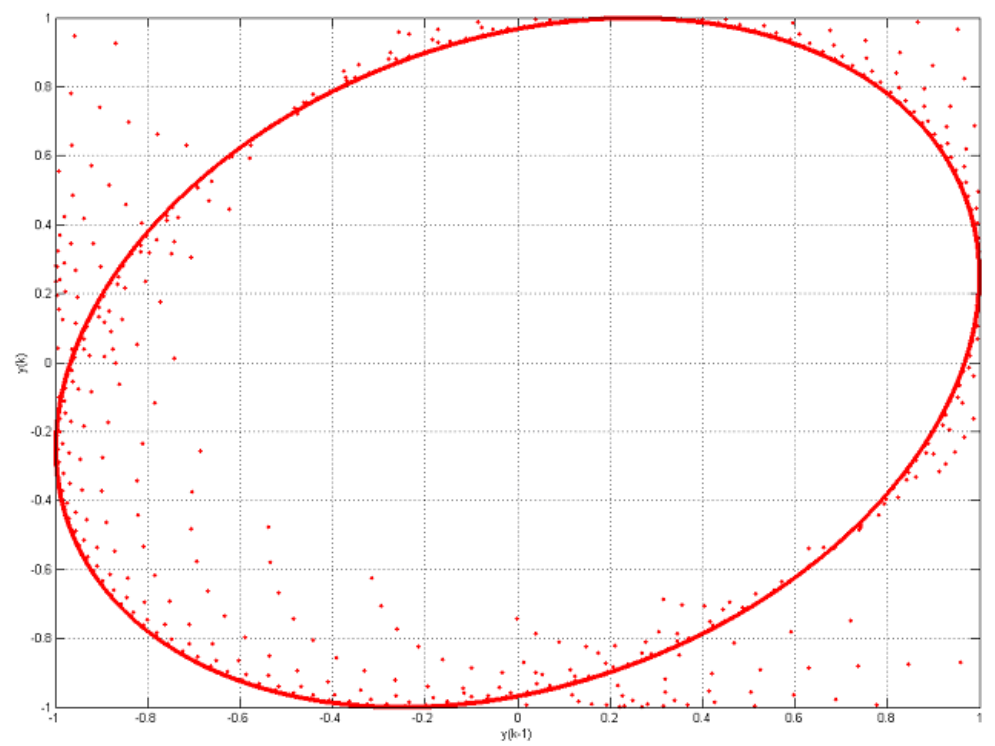

FIG. 4a. The plot of $y(k-1)$ versus $y(k)$ for type I trajectory with $c=0.99, a=0.5$ and $\mathbf{x}(0)=\left[\begin{array}{c}0.612 \\ -0.612 \\ 0.3\end{array}\right]$.

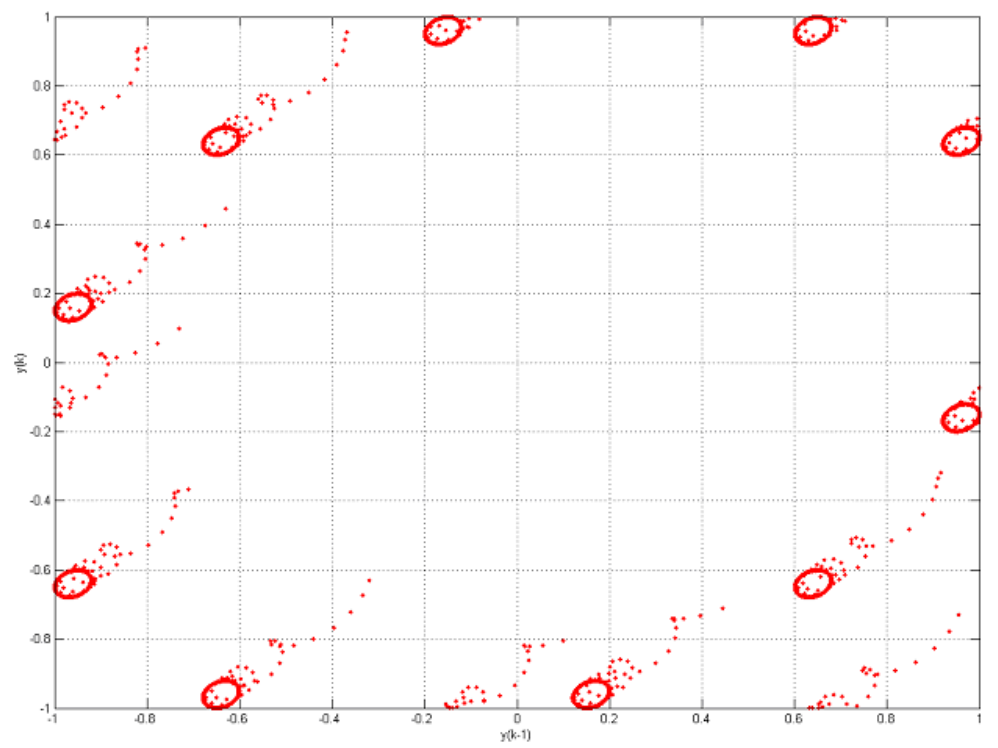

FIG. 4b. The plot of $y(k-1)$ versus $y(k)$ for type II trajectory with $c=0.99, a=0.5$ and $\mathbf{x}(0)=\left[\begin{array}{c}0.616 \\ -0.616 \\ 0.3\end{array}\right]$. 


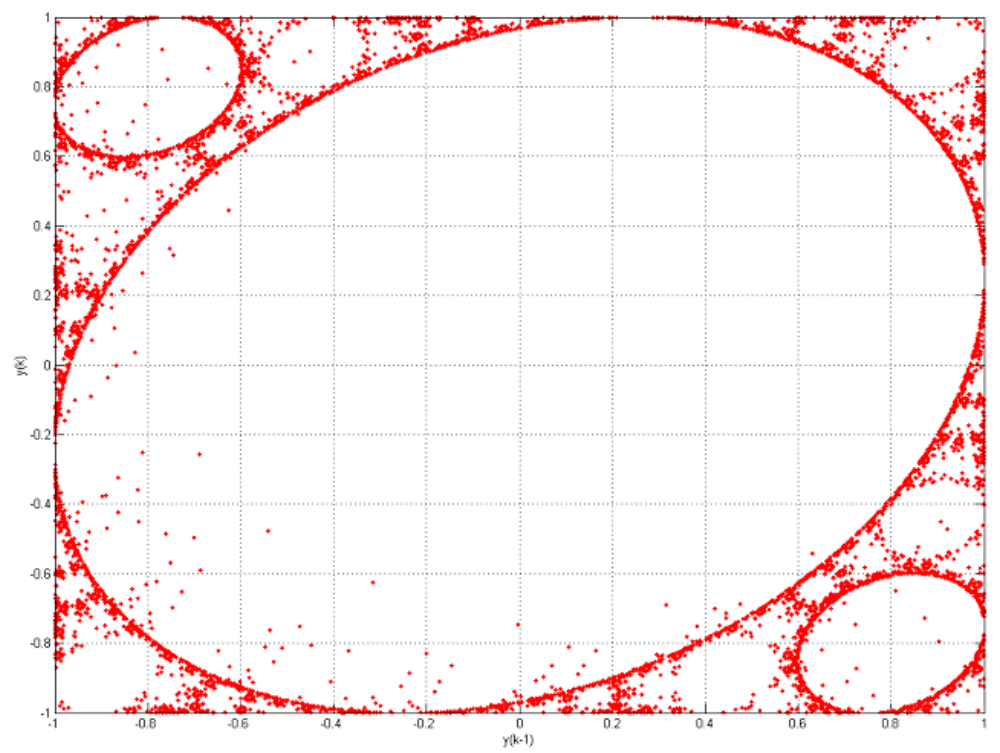

FIG. 4c. The plot of $y(k-1)$ versus $y(k)$ for type III trajectory with $c=0.99, a=0.5$ and $\mathbf{x}(0)=\left[\begin{array}{c}0.6135 \\ -0.6135 \\ 0.3\end{array}\right]$.

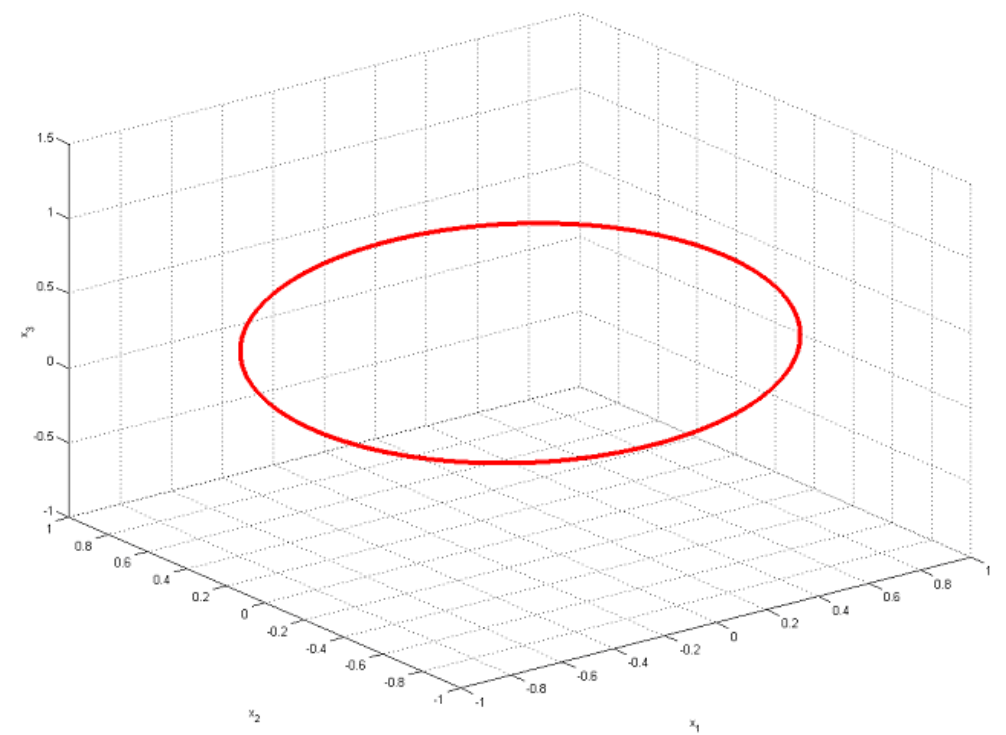

FIG. 5a. Type I trajectory with $c=1, a=0.5$ and $\mathbf{x}(0)=\left[\begin{array}{c}0.612 \\ -0.612 \\ 0.3\end{array}\right]$. 


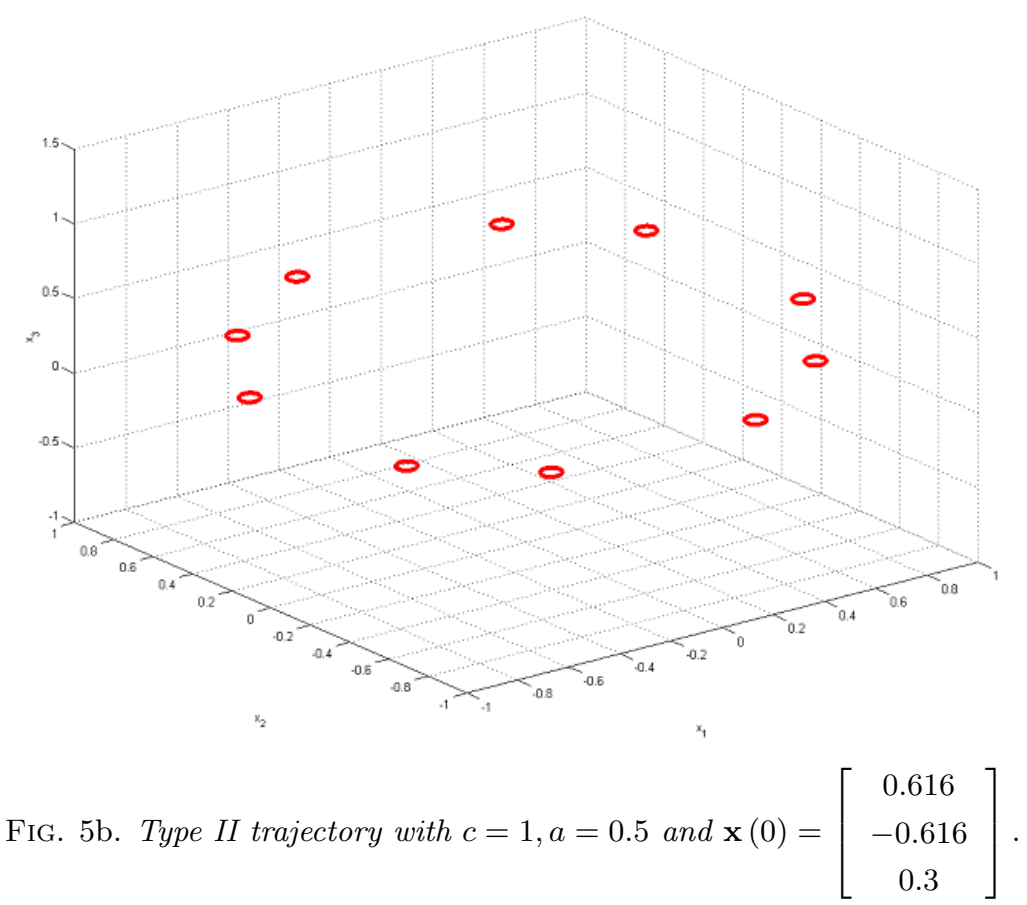

or $y_{1}(k)+x_{3}(0) \geq 1$ and $y_{1}(k-1)+x_{3}(0) \geq 1$.

As there are three types of trajectories for the corresponding second-order subsystem, there are also three types of trajectories for the plot of $y(k-1)$ versus $y(k)$, as shown in figures $7 \mathrm{a}, 7 \mathrm{~b}$ and $7 \mathrm{c}$, respectively.

4.3. $c=-1$.

For $c=-1$, the output of the first-order subsystem gives oscillatory output, while that of the corresponding second-order digital filter may exhibit oscillating, limit cycle or chaotic behaviors. Hence, the output of the third-order digital filter may be more complicated.

When $c=-1$, since $u(k)=0$ for $k \geq 0$, if $x_{3}(0) \neq-1$, we have:

$$
\text { and } x_{3}(k)=(-1)^{k} \cdot x_{3}(0) \text { for } k \geq 0 \text {. }
$$

If $x_{3}(0)=-1$, we have:

$$
x_{3}(k)=-1 \text { for } k \geq 0 .
$$

Since

$$
y_{2}(k)=x_{3}(k) \text { for } k \geq 0,
$$

if $x_{3}(0) \neq-1$, the state trajectories are on two phase planes with $x_{3}=x_{3}(0)$ or $x_{3}=-x_{3}(0)$, as shown in figure 8. In this case, there are no attractive or repelling 

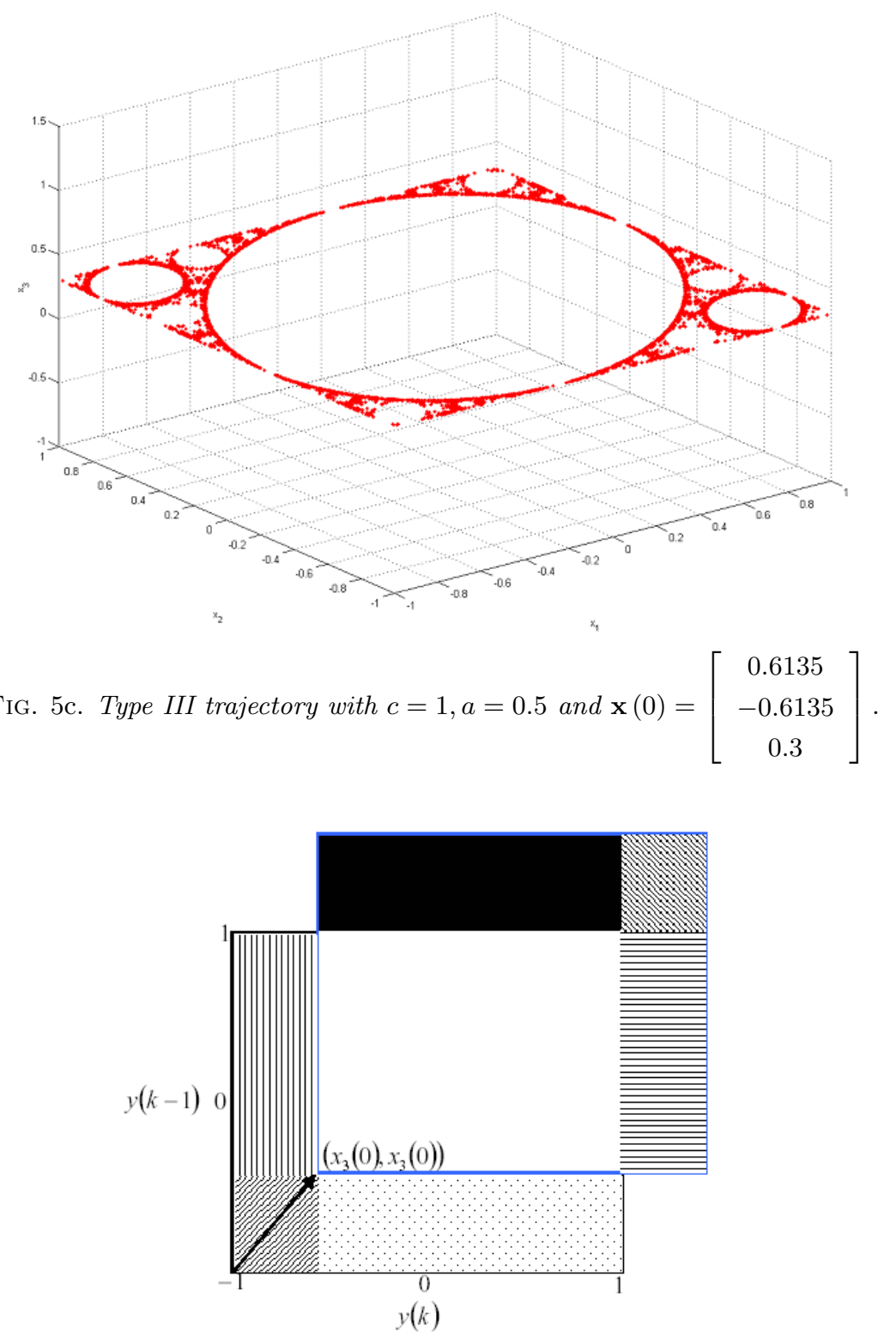

FIG. 6. Diagram showing how adding a constant value with two's complement to a signal.

regions for the trajectories. Similarly, there are three types of trajectories for the third-order digital filter, depending on the initial conditions of the corresponding second-order digital filter, as shown in figures $8 \mathrm{a}, 8 \mathrm{~b}$ and $8 \mathrm{c}$, respectively. If the system exhibits type I or type II trajectories, the trajectories on the elliptical orbits of the planes are dense if $\theta$ is not a rational multiple of $\pi$. For the type III trajectory, the trajectory on the fractal region of the planes is also dense. If $x_{3}(0)=-1$, the state trajectory is on a phase plane with $x_{3}=-1$, and the result is similar to that 


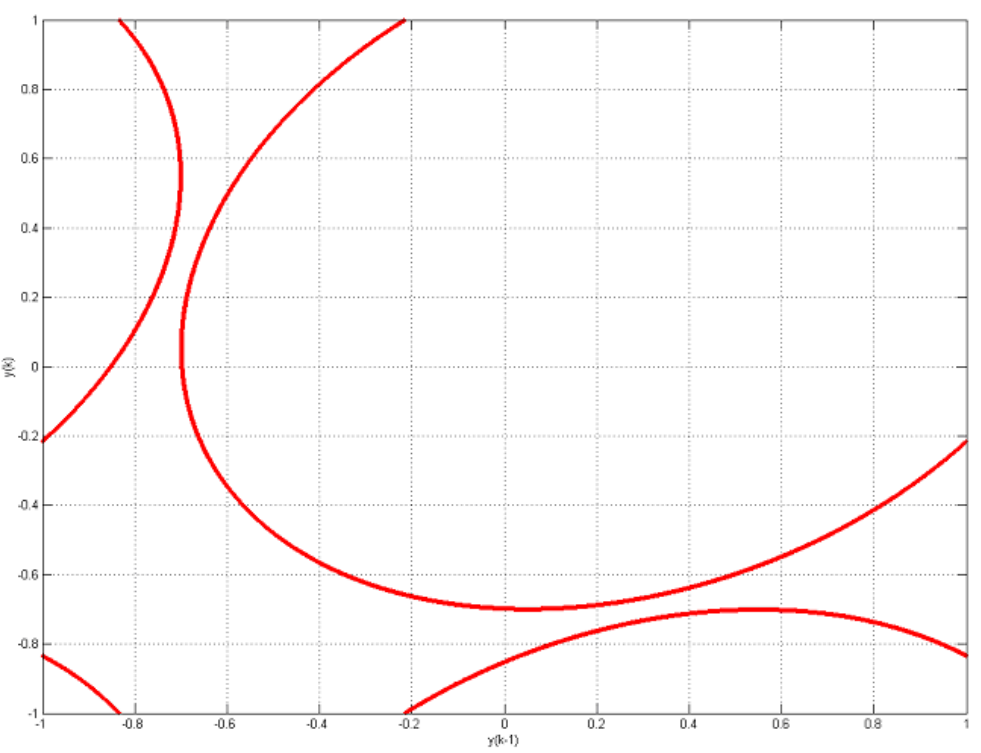

FIG. 7a. The plot of $y(k-1)$ versus $y(k)$ for type I trajectory with $c=1, a=0.5$ and $\mathbf{x}(0)=\left[\begin{array}{c}0.612 \\ -0.612 \\ 0.3\end{array}\right]$.

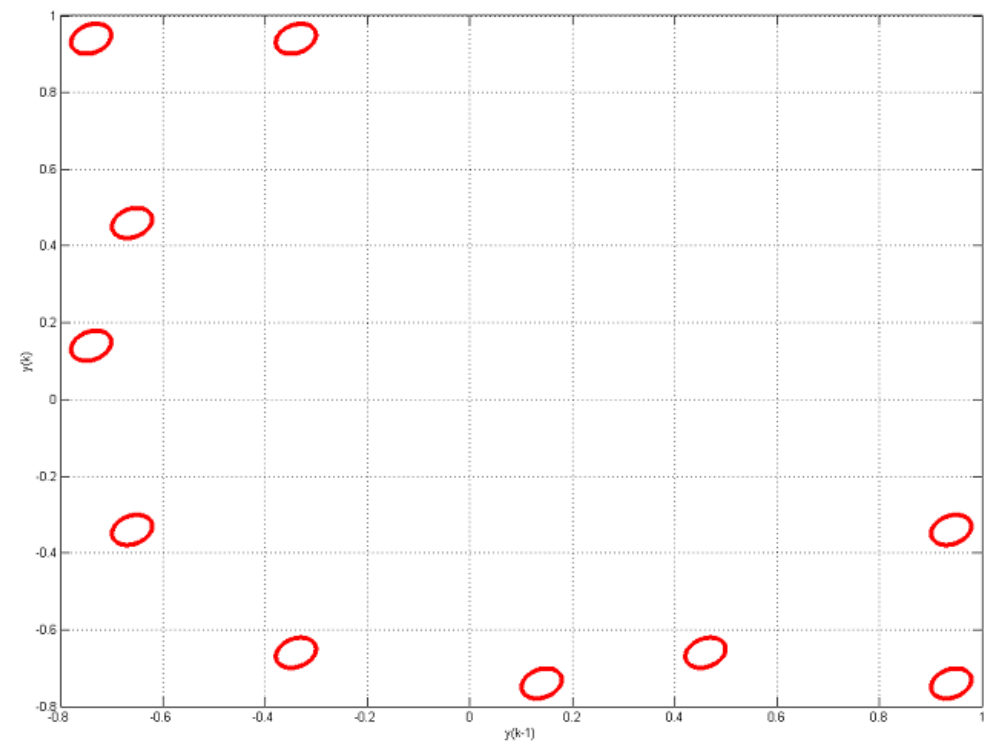

FIG. 7b. The plot of $y(k-1)$ versus $y(k)$ for type II trajectory with $c=1, a=0.5$ and $\mathbf{x}(0)=\left[\begin{array}{c}0.616 \\ -0.616 \\ 0.3\end{array}\right]$. 


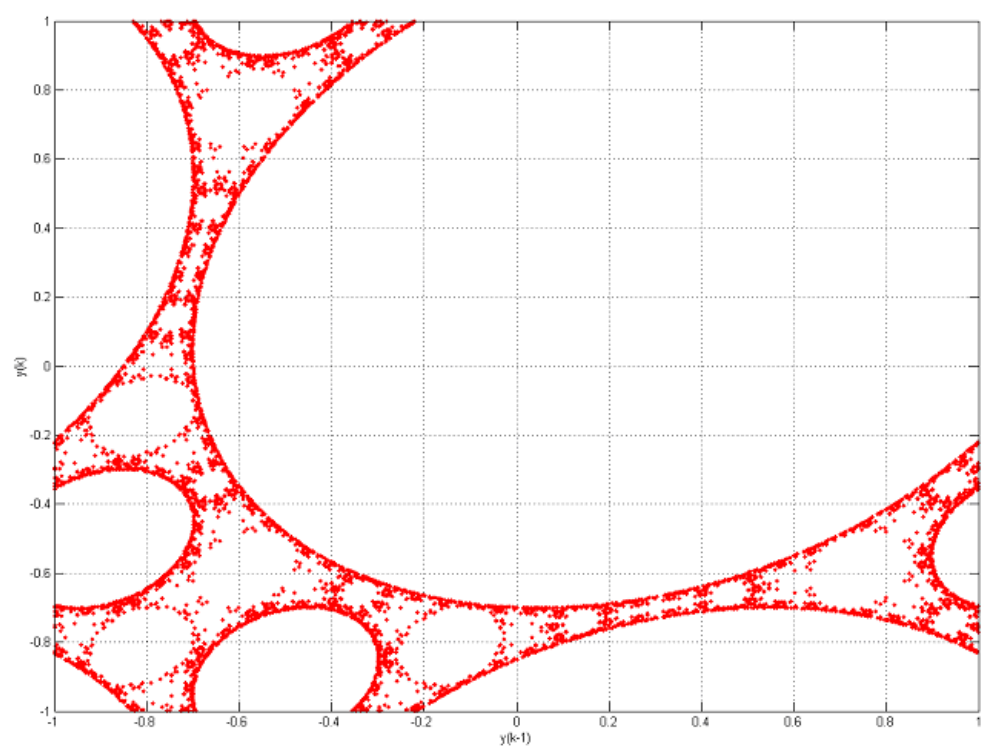

FIG. 7c. The plot of $y(k-1)$ versus $y(k)$ for type III trajectory with $c=1, a=0.5$ and $\mathbf{x}(0)=\left[\begin{array}{c}0.6135 \\ -0.6135 \\ 0.3\end{array}\right]$.

discussed in section 4.2 .

As $y_{1}(k)=x_{1}(k)$ for $k \geq 0$, if $x_{3}(0) \neq-1$, then $y_{2}(k)=x_{3}(0)$ and $y(k)=$ $f\left(y_{1}(k)+x_{3}(0)\right)$ for $k$ is even, and $y_{2}(k)=-x_{3}(0)$ and $y(k)=f\left(y_{1}(k)-x_{3}(0)\right)$ for $k$ is odd. Since the plot of $y(k-1)$ versus $y(k)$ can be obtained by applying the two's complement arithmetic operation on the shifted trajectory of the corresponding second-order digital filter both horizontally and vertically, the diagram involves shifting both $x_{3}(0)$ and $-x_{3}(0)$ in both horizontal and vertical directions. Hence, the corresponding modified trajectories are overlapped, as shown in figure 9. As there are three types of trajectories for the corresponding second-order subsystem, there are also three types of trajectories for the plot of $y(k-1)$ versus $y(k)$, as shown in figures $9 \mathrm{a}, 9 \mathrm{~b}$ and $9 \mathrm{c}$, respectively.

5. Discussion. As explained in section 4, there is a one-to-one correspondence mapping of the types of behavior of the third-order and the corresponding secondorder digital filter. Therefore many of our results in [11] and other interesting results in [15] apply.

5.1. When the corresponding first-order subsystem is stable or marginally stable and the corresponding second-order subsystem is marginally stable, the finite state machine implementation of the digital filter may behave in a near-chaotic way even when its corresponding infinite-state machine implementation does not exhibit chaotic behaviors [11]. 


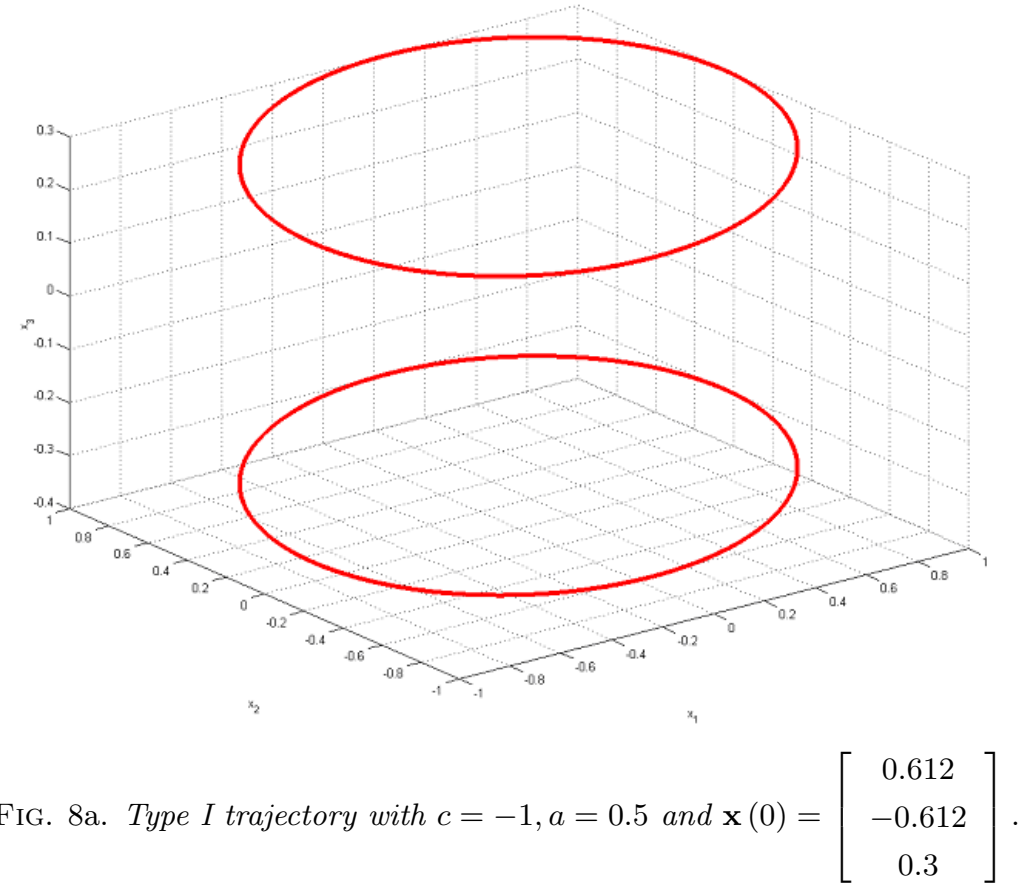

5.2. If the corresponding first-order and second-order subsystem is implemented in a normal form, then the nonlinear behaviors, such as limit cycles and chaotic behavior, will not occur [15]. However, more calculations may be performed by the filter. This is because there is only one addition, one multiplication and two delays for the direct form realization if $b=-1$, but two additions, two multiplications and two delays for the normal form realization.

5.3. In order to measure the similarity of the trajectories of the second-order subsystem and the plot of the output of the third-order system $y(k-1)$ versus $y(k)$, we can treat these two plots as two images, $\mathbf{X}$ and $\mathbf{Y}$, respectively, where $\mathbf{X}$ is the image corresponding to the phase portrait of the second-order subsystem and $\mathbf{Y}$ is the image corresponding to the plot of the output of the third-order system $y(k-1)$ versus $y(k)$. When $c=1, \mathbf{Y}=f_{1}\left(\mathbf{X}+\mathbf{X}_{0}\right)$, where $\mathbf{X}_{0}$ corresponds to the shift due to the initial condition of the first-order subsystem and $f_{1}$ corresponds to the two-dimensional two's complement operation defined in section 4.2. Since these operations are just row operations and column operations, there exists a matrix $\mathbf{T}_{\mathbf{X}_{0}}=\left[\begin{array}{ll}\mathbf{0}_{(M-n) x n} & \mathbf{I}_{(M-n) x(M-n)} \\ \mathbf{I}_{n x n} & \mathbf{0}_{n x(M-n)}\end{array}\right]$ such that $\mathbf{Y}=\mathbf{T}_{\mathbf{X}_{0}} \cdot \mathbf{X} \cdot \mathbf{T}_{\mathbf{X}_{0}}$. Hence, we can investigate the feasibility of using this index $n$, where $0 \leq n<\frac{M}{2}$, as a measure for the similarity between these trajectories. A similar approach can be adopted to study the case when $c=-1$. 


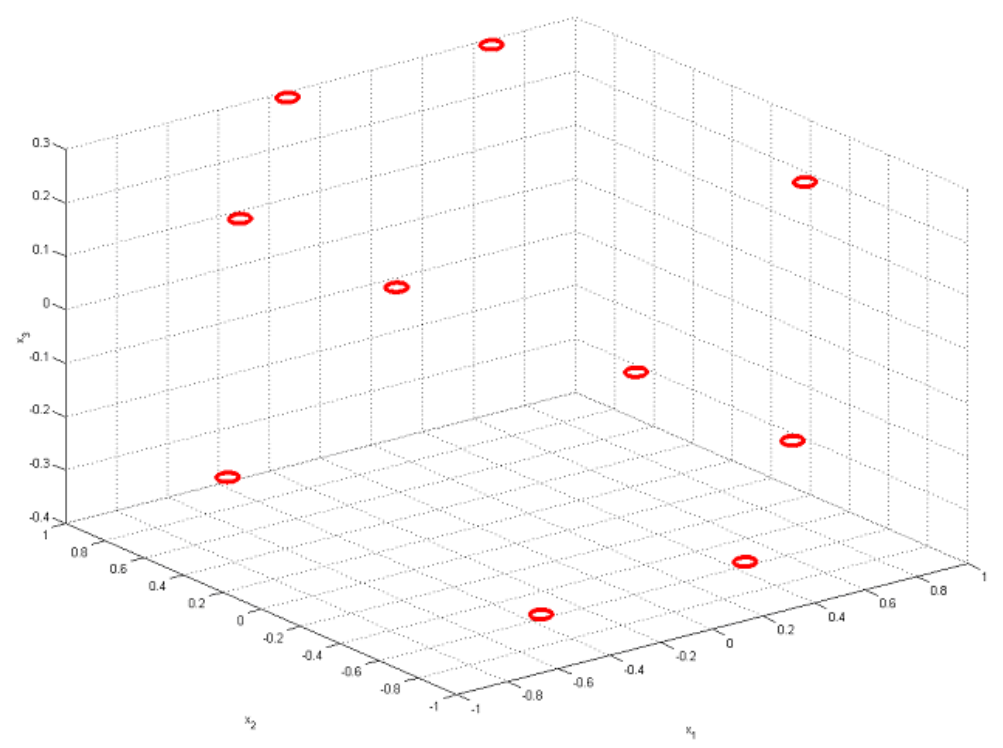

FIG. 8b. Type II trajectory with $c=-1, a=0.5$ and $\mathbf{x}(0)=\left[\begin{array}{c}0.616 \\ -0.616 \\ 0.3\end{array}\right]$.

6. Conclusion. When a third-order digital filter with two's complement arithmetic is realized in a parallel form, the output of the third-order digital filter may exhibit random like chaotic or limit cycle behaviors. However, the plot of $y(k-1)$ versus $y(k)$ shows some regular patterns, which is similar to trajectories of the autonomous response of a second-order digital filter, as shown in figures 2, 4, 7 and 9. If the corresponding second-order digital filter exhibits type I trajectory, then the diagram also shows a type I trajectory, similarly for the type II and type III trajectories, even though overflow may occur when the output of the first-order digital filter is added to that of the corresponding second-order digital filter. When the pole of the first-order subsystem equals to one, the plot of $y(k-1)$ versus $y(k)$ exhibits the state trajectory of the corresponding second-order digital filter shifted by the two's complement operation. The shift only depends on the initial condition of the corresponding first-order digital filter. Similarly, when the pole of the corresponding first-order digital filter equals to minus one, the plot of $y(k-1)$ versus $y(k)$ shows the overlap of two state shifted trajectories of the corresponding second-order digital filter modifed by the two's complement operation. Again, the shift only depends on the initial condition of the corresponding first-order digital filter.

Acknowledgement. The work described in this paper was substantially supported by The Hong Kong Polytechnic University. 


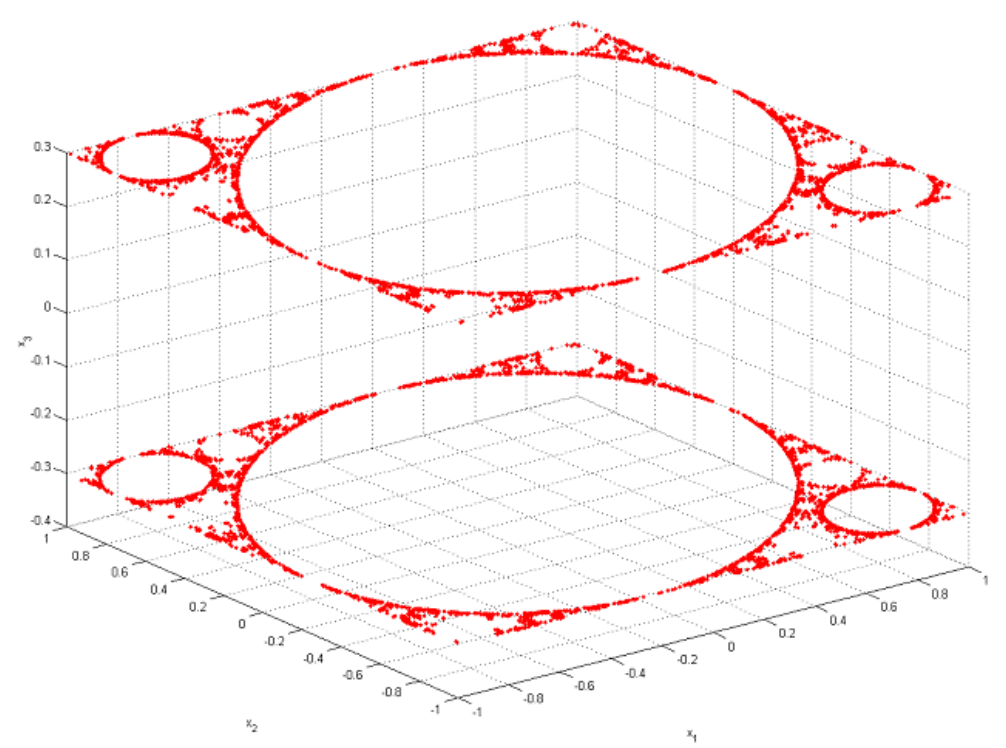

FIG. 8c. Type III trajectory with $c=-1, a=0.5$ and $\mathbf{x}(0)=\left[\begin{array}{c}0.6135 \\ -0.6135 \\ 0.3\end{array}\right]$.

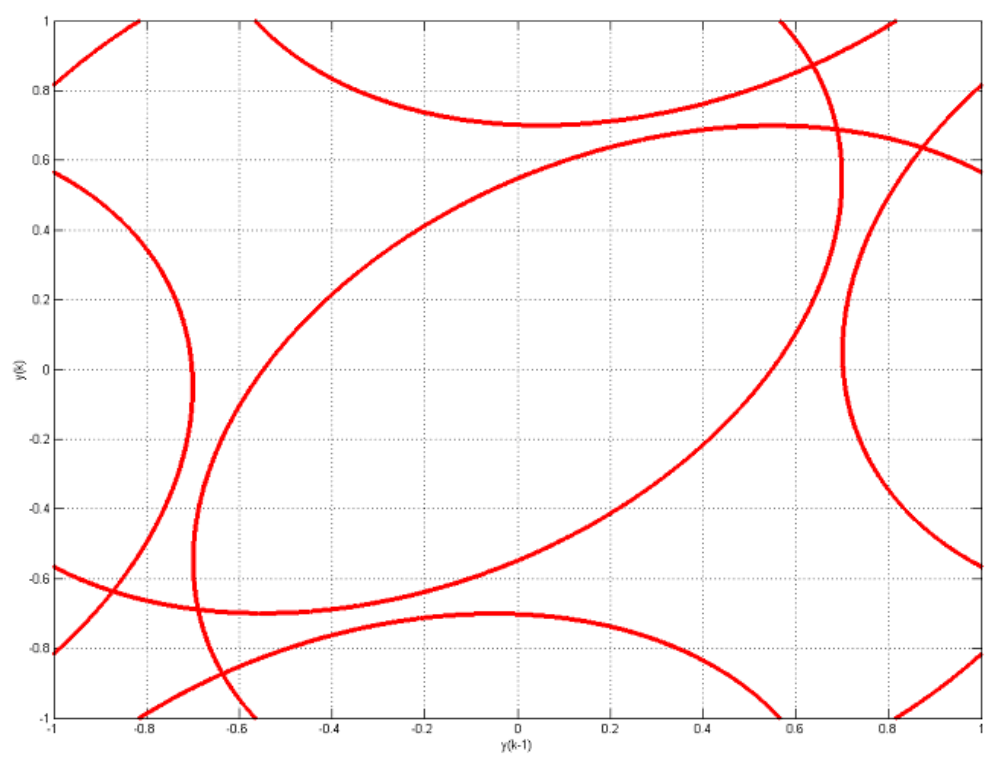

FIG. 9a. The plot of $y(k-1)$ versus $y(k)$ for type I trajectory with $c=-1, a=0.5$ and $\mathbf{x}(0)=\left[\begin{array}{c}0.612 \\ -0.612 \\ 0.3\end{array}\right]$. 


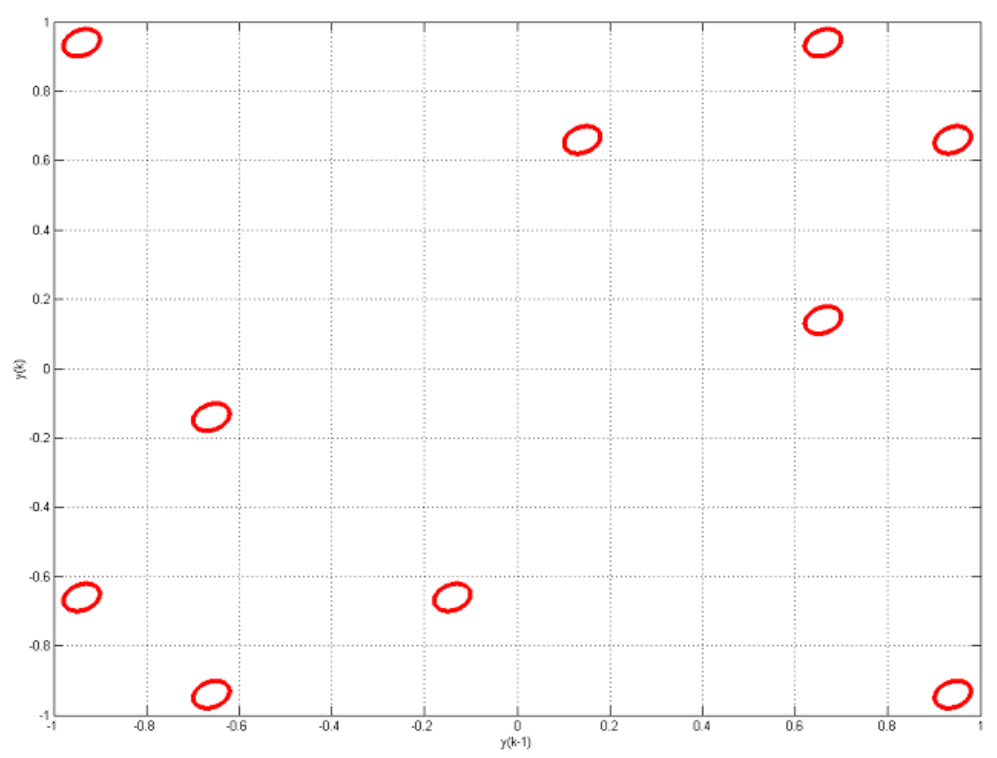

FIG. 9b. The plot of $y(k-1)$ versus $y(k)$ for type II trajectory with $c=-1, a=0.5$ and $\mathbf{x}(0)=\left[\begin{array}{c}0.616 \\ -0.616 \\ 0.3\end{array}\right]$.

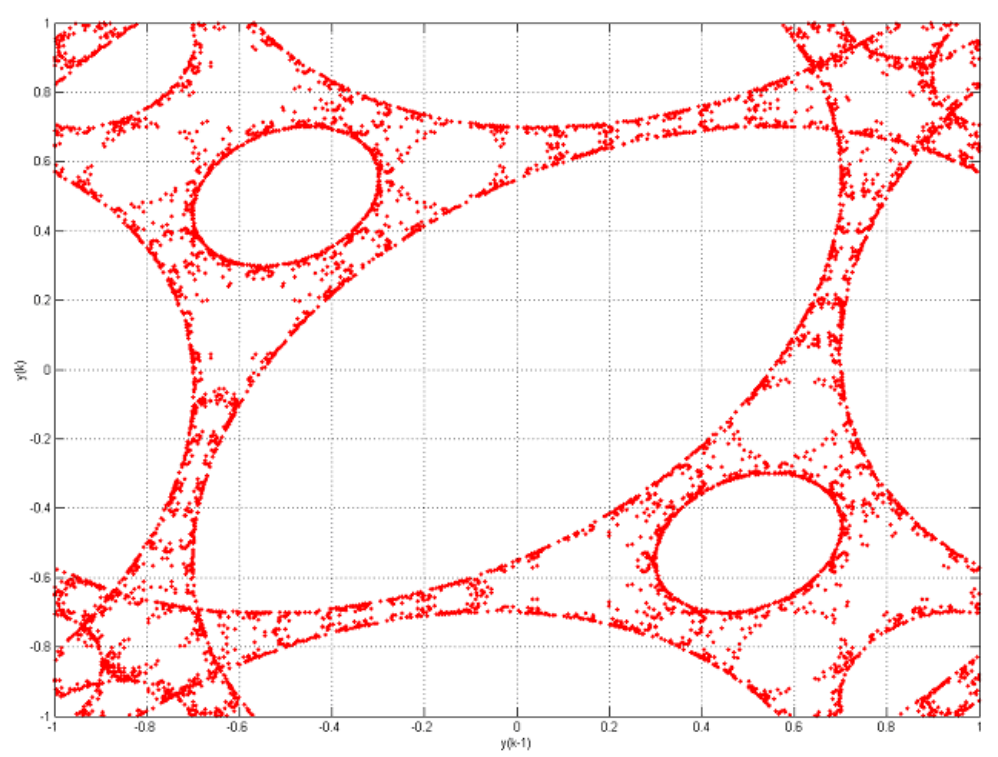

FIG. 9c. The plot of $y(k-1)$ versus $y(k)$ for type III trajectory with $c=-1, a=0.5$ and $\mathrm{x}(0)=\left[\begin{array}{c}0.6135 \\ -0.6135 \\ 0.3\end{array}\right]$. 


\section{REFERENCES}

[1] L. O. Chua And T. Lin, Chaos in digital filters, IEEE Transactions on Circuits and Systems, 35:6(1988), pp. 648-658.

[2] Z. Galias And M. J. Ogorzalek, Bifurcation phenomena in second-order digital filter with saturation-type adder overflow characteristic, IEEE Transactions on Circuits and Systems, 37:8(1990), pp. 1068-1070.

[3] L. O. ChuA AND T. Lin, Chaos and fractals from third-order digital filters, International Journal of Circuit Theory and Applications, 18(1990), pp. 241-255.

[4] L. O. ChuA AND T. Lin, Fractal pattern of second-order non-linear digital filters: a new symbolic analysis, International Journal of Circuit Theory and Applications, 18(1990), pp. $541-550$.

[5] T. Lin And L. O. ChuA, On chaos of digital filters in the real world, IEEE Transactions on Circuits and Systems, 38:5(1991), pp. 557-558.

[6] Z. Galias and M. J. Ogorzalek, On symbolic dynamics of a chaotic second-order digital filter, International Journal of Circuit Theory and Applications, 20(1992), pp. 401-409.

[7] C. W. Wu ANd L. O. ChuA, Properties of admissible symbolic sequences in a second-order digital filter with overflow non-linearity, International Journal of Circuit Theory and Applications, 21(1993), pp. 299-307.

[8] L. Kocarev and L. O. Chua, On chaos in digital filters: case $b=-1$, IEEE Transactions on Circuits and Systems II: Analog and Digital Signal Processing, 40:6(1993), pp. 404-407.

[9] L. Kocarev, C. W. Wu, And L. O. Chua, Complex behavior in digital filters with overflow nonlinearity: analytical results, IEEE Transactions on Circuits and Systems II: Analog and Digital Signal Processing, 43:3(1996), pp. 234-246.

[10] X. Yu and Z. Galias, Periodic behaviors in a digital filter with two's complement arithmetic, IEEE Transactions on Circuits and Systems I: Fundamental Theory and Applications, 48:10(2001), pp. 1177-1190.

[11] W. K. Ling, F. C. K. LuK, AND P. K. S. TAM, Further investigation on chaos of real digital filters, to appear in International Journal of Bifurcation and Chaos.

[12] W. K. Ling AND P. K. S. TAM, Some new trajectory patterns and periodic behaviors of unstable second-order digital filter with two's complement arithmetic, to appear in International Journal of Bifurcation and Chaos.

[13] W. K. Ling, P. K. S. TAM, AND X. YU, Step response of a second-order digital filter with two's complement arithmetic, favourite comments from reviewers of the IEEE Transactions on Circuits and Systems I: Fundamental Theory and Applications.

[14] W. K. Ling AND P. K. S. TAm, Sinusoidal response of a second-order digital filter with two's complement arithmetic, favourite comments from reviewers of the IEEE Transactions on Circuits and Systems I: Fundamental Theory and Applications.

[15] Guo Fang Xu and Tamal Bose, Elimination of limit cycles due to two's complement quantization in normal form digital filters, IEEE Transactions on Signal Processing, 45:12(1997), pp. 2891-2895. 